\title{
Map2k5-Deficient Mice Manifest Phenotypes and Pathological Changes of Dopamine Deficiency in the Central Nervous System
}

\author{
Yumeng Huang ${ }^{11}$, Pei Wang ${ }^{1 t}$, Rodrigo Morales ${ }^{2,3}$, Qi Luo ${ }^{1}$ and Jianfang Ma ${ }^{1 *}$ \\ ' Department of Neurology, Institute of Neurology, Shanghai Jiao Tong University Medical School Affiliated Ruijin Hospital, \\ Shanghai, China, ${ }^{2}$ Department of Neurology, McGovern Medical School, The University of Texas Health Science Center \\ at Houston, Houston, TX, United States, ${ }^{3}$ Centro Integrativo de Biologia y Quimica Aplicada (CIBQA), Universidad Bernardo \\ O'Higgins, Santiago, Chile
}

OPEN ACCESS

Edited by:

Benoit Laurent

Université de Sherbrooke, Canada

Reviewed by:

Cathy Tournier,

The University of Manchester,

United Kingdom

Sebastien Carnicella,

INSERM U1216 Grenoble Institut des

Neurosciences (GIN), France

*Correspondence:

Jianfang Ma

mif10924@rih.com.cn

tThese authors share first authorship

Received: 10 January 2021 Accepted: 03 May 2021

Published: 08 June 2021

Citation: Huang $Y$, Wang $P$, Morales $R$, Luo Q and Ma J (2021)

Map2k5-Deficient Mice Manifest

Phenotypes and Pathological Changes of Dopamine Deficiency

in the Central Nervous System.

Front. Aging Neurosci. 13:651638.

doi: 10.3389/fnagi.2021.651638
MAP2K5, a member of the MAPK family, is associated with central nervous system disorders. However, neural functional of Map2 $k 5$ from animal models were not well examined so far. Here, we established a Map2k5-targeted knockout mouse model to investigate the behavior phenotypes and its underlying molecular mechanism. Our results showed that female Map2k5 mutant mice manifested decreased circadiandependent ambulatory locomotion, coordination, and fatigue. Male Map2k5 mutant mice displayed impairment in open field exploration and prepulse inhibition of acoustic startle response (ASR) when compared with wild-type controls. Furthermore, Map2k5 mutant mice showed a decreased dopaminergic cell survival and tyrosine hydroxylase levels in nigrostriatal pathway, indicating a crucial role of MAP2K5 in regulating dopamine system in the central nervous system. In conclusion, this is the first study demonstrating that Map2k5 mutant mice displayed phenotypes by disturbing the dopamine system in the central nervous system, implicating Map2k5 mutant mouse as a promising model for many dopamine related disorders.

Keywords: MAP2K5, mouse model, dopamine, sensorimotor phenotypes, restless legs syndrome, Parkinson's disease

\section{INTRODUCTION}

MAP2K5 (mitogen-activated protein kinase kinase 5, also known as MEK5) has been associated with various diseases including cardiovascular diseases, cancers, and central nervous system disorders (Appari et al., 2017; Liu et al., 2017; Castro et al., 2019). In the central nervous system, some polymorphisms of MAP2K5 have been associated with restless legs syndrome/William-Ekbom disease (RLS/WED) and neuropsychologic disorders (Winkelmann et al., 2007; Yang et al., 2011; Li et al., 2017). In addition, studies also suggested

Abbreviations: ACh, acetylcholine; $\mathrm{AMB}$, ambulatory activity; ASR, acoustic startle response; DA, dopamine; Epi, epinephrine; GAPDH, glyceraldehyde-3-phosphate dehydrogenase; Gln, glutamine; HCG, human chorionic gonadotropin; HPLC, high-performance liquid chromatography; KO, knockout; MAP2K5, mitogen-activated protein kinase kinase 5; P18d, postnatal 18 days; PBS, phosphate-buffered saline; PFA, paraformaldehyde; Phe, phenylalanine; PLMS, periodic limb movements; PPI, prepulse inhibition; qRT-PCR, semi-quantitative reverse transcriptase-PCR; RLS/WED, restless legs syndrome/Willis-Ekbom disease; SNc, substantia nigra pars compacta; SNP, single-nucleotide polymorphism; TH, tyrosine hydroxylase; TOT, total activity; Tyr, tyrosine; WT, wild type. 
its etiologic or therapeutic role in amyotrophic lateral sclerosis, Parkinson's disease (PD), schizophrenia, suicidal behavior, and others (Gan-Or et al., 2015; Jo et al., 2017; Kang et al., 2018; Cabrera-Mendoza et al., 2020). Although the role of Map2k5Erk5 in cardiovascular diseases and cancer has been mostly clarified, the molecular mechanism of Map2 $\mathrm{k} 5$ in the central nervous system remained largely unknown.

MAP2K5 is a member of the MAP kinase family, and it is involved in growth factor-induced cell proliferation, survival, and differentiation (Dinev et al., 2001; Liu et al., 2006). It specifically activates ERK5 by phosphorylation on threonine and tyrosine residues (Johnson and Lapadat, 2002). The MAP2K5-ERK5 pathway regulates many transcription factors including MEF2, c-MYC, and NF-кB (English et al., 1998; Kato et al., 2000; Simões et al., 2015), which were identified as essential in cells' survival and development. Moreover, the role of the MAP2K5-ERK5 pathway in neurons' proliferation, differentiation, and apoptosis were demonstrated in many studies, and included activity in cortical, dopaminergic, and GABAergic neurons (Liu et al., 2006; Zou et al., 2012; Parmar et al., 2015; Ding et al., 2020). Target deletion of MAP2K5 in animals may help us to discover its role in biochemical processes and gross functions. However, there are few studies using animal models focused on the role of MAP2K5 in the central nervous system. This is probably due to the fact that fully knockout Map2k5 mice died at early embryonic periods from heart deficits (Wang et al., 2005).

Based on the finding that polymorphisms (rs12593813, rs11635424, and rs868036) in MAP2K5 were associated with a reduced risk of RLS/WED, a neurological disorder characterized by dopamine and iron dysfunction, we established a targeted deletion of Map2k5 in mice. Specifically, we focused on the deletion of exons 18-22, which harbor three risky SNPs. This model was hypothesized to be useful in exploring the role of this protein in the central nervous system in vivo. We found that the homozygous Map2k5-deficient mouse died at mid gestation, similarly, as previously reported (Wang et al., 2005). Heterozygous female Map2k5-deficient mouse manifested decreased locomotion, impaired coordination, and was prone to fatigue. Interestingly, these phenotypes are contrary to RLS/WED and, in some aspects, similar to PD (another movement disorder involving dopamine system disturbance). Furthermore, we found reduced dopaminergic neurons and decreased tyrosine hydroxylase (TH) levels in female Map2k5-deficient mice compared with wild-type (WT) mice in nigrostriatal dopaminergic pathway, which may explain the motor dysfunction in female Map2k5-deficient mice. To our knowledge, this is the first in vivo study that demonstrated the relationship of dopaminergic system disturbance with the Map2k5-Erk5 pathway.

\section{MATERIALS AND METHODS}

\section{Map2k5 ${ }^{\mathrm{em} 41} \mathrm{Cd} 71454$ Mice}

Animal experiments were performed in accordance with the guidelines and policies of the Association for Assessment and Accreditation of Laboratory Animal Care International
(AAALAC International), China National Accreditation Service for Conformity Assessment (CNAS), and Laboratory Animal Resource Center of Shanghai Jiao Tong University School of Medicine. Map2k $5^{\mathrm{em} 41} \mathrm{Cd} 71454$ mice were created in Nanjing, China (GemPharmmatech Co., Ltd.) via CRISPR/Cas9 technique on a C57B6/JGpt (GemPharmmatech Co., Ltd.) background. In this strain, based on the findings that most reported SNPs in MAP2k5 of RLS/WED [rs12593813 (A/G), rs11635424 $(\mathrm{A} / \mathrm{G})$, and $\mathrm{rs} 868036(\mathrm{~T} / \mathrm{A})]$ were located in the intron area of gene Map2k5 between exons 17-18 and exons 19-20 (RefSeq: NM_011840), transgenic mice with Map2k5 exons 18-22 deletion were constructed. Two sgRNAs on both sides of the targeted region were designed specifically (Figure 1A) (sgRNA1: GGCTCCGTAAGTGTCCACATGGG; sgRNA3: TGCAGGCACGGGCCTTTGACTGG) and constructed by using MEGAshortscript ${ }^{\mathrm{TM}}$ Kit (Thermo Fisher Scientific, Waltham, MA, United States). In order to microinject Cas9 protein and sgRNA into fertilized eggs, male C57BL6/J mice were individually caged for 1 week before mating. Female C57BL6/J mice were given $5 \mathrm{IU}$ of serum gonadotropin and 5 IU of human chorionic gonadotropin (HCG) to pregnant horses for superovulation. After C57BL6/J male and female mice were injected with HCG, the fertilized eggs of male and female mice were taken for mating. SgRNA (40 ng/ $\mu \mathrm{l})$ and Cas9 protein (40 $\mathrm{ng} / \mu \mathrm{l})$ were injected into the cytoplasm of the fertilized egg at the single-cell stage using continuous flow injection microinjection. The surviving two-cell embryos were implanted into the fallopian tube of a pseudo-pregnant female. The generated mice (F0) were analyzed in the born pups. F0 mice were mated with C57BL6/J wild-type mice to gain the obtained F1 mice. Their Map2k5 with exons 18-22 deletion sequencing were examined by Sanger Sequencing (Supplementary Material 1). To exclude the off-target effect, genetic sequencing of high frequent loci of each sgRNA (sgRNA1 and sgRNA3) were predicted by CRISPOR (Concordet and Haeussler, 2018) ${ }^{1}$ and confirmed by Sanger sequencing (Supplementary Material 2).

All offspring were identified by genotyping tail DNA (Accili et al., 1996). KO (knockout) primers (5' GATGAA GCAAAGGGTGACGATAG $3^{\prime}$ and $5^{\prime}$ CTTGGGAAGGCAAGC ACTACTC $\left.3^{\prime}, 940 \mathrm{bp}\right)$ and WT primers (5' ACATTCCA TCCTACCTCAGCCTAG $3^{\prime}$ and 5' CCTACTTGCTTGGCTTCA GTCTG 3', 461 bp) were used for this purpose (Figure 1B). F1 mice were interbred to obtain F2 homozygous, while homozygous mutants were examined dead prenatally after. Large quantities of F3 experiment's mice were interbred with F2 heterozygous mice (some PCR genetic identification results are shown in Figure 1C). In all experiments, heterozygous Map2 $\mathrm{k}^{+/-}$mice and their WT littermates were used, as homozygotes were embryonic lethal.

\section{mRNA Prepared and Semiquantitative Reverse Transcriptase -PCR}

Total RNA was extracted from homogenized frozen brain, heart, and spleen tissue followed by TRIzol ${ }^{\mathrm{TM}}$ Reagent (Invitrogen, Carlsbad, CA, United States) extraction protocols. Reverse

\footnotetext{
${ }^{1}$ http://crispor.tefor.net
} 
A

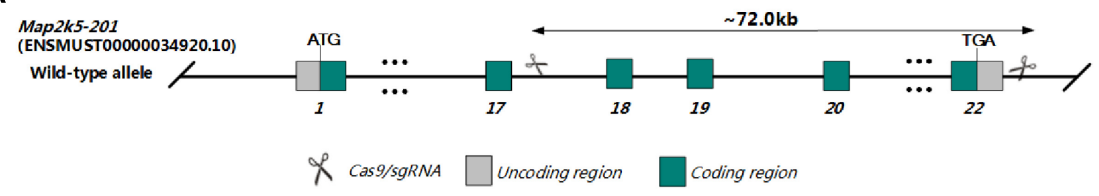

B

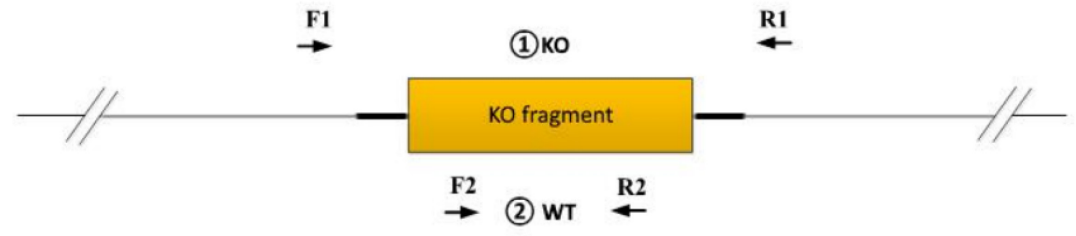

Primer (1) (2) PCR Reaction Number - Sequencing Region

C

FIGURE 1 | (A) Targeting strategy of Map2k5-targeted allele. Green boxes represent coding regions, gray boxes represent noncoding exon regions, and scissors represent the targeted area of Map2k5 between exons 18 and 22. (B) Primers designed for genotype identification of Map2k5+/ - heterozygous and wild-type (WT) mice. (C) PCR results of some Map2k5 ${ }^{\mathrm{em} 41} \mathrm{Cd} 71454$ mice.

transcription was performed using PrimeScript ${ }^{\mathrm{TM}}$ RT reagent Kit with gDNA Eraser [TAKARA Biomedical Technology (Beijing) Co., Ltd., China] and qRT-PCR using TB Green ${ }^{\circledR}$ Premix Ex $\mathrm{Taq}^{\mathrm{TM}}$ II [TAKARA Biomedical Technology (Beijing) Co., Ltd., China] with the SYBR Green Method (Applied Biosystems, Foster City, CA, United States). Oligonucleotides_Map2k5 (5' AAG CGA TGA AGA GAT GAA G $3^{\prime}$ and $5^{\prime}$ ATG TAT GTT CCG TTC CCC A $3^{\prime}$ ) targeted the dominant Map2k5 mRNA. The primers of the glyceraldehyde-3-phosphate dehydrogenase (Gapdh) gene (5' AAA TGG TGA AGG TCG GTG TG 3' and $5^{\prime}$ AGG TCA ATG AAG GGG TCG TT $3^{\prime}$ ) were used as the reference gene-glyceraldehyde-3-phosphate dehydrogenase (Gapdh). Run method: (a) $30 \mathrm{~s}$ at $95^{\circ} \mathrm{C}$; (b): 40 cycles of $5 \mathrm{~s}$ at $95^{\circ} \mathrm{C}, 34 \mathrm{~s}$ at $64^{\circ} \mathrm{C}$; (c) $15 \mathrm{~s}$ at $95^{\circ} \mathrm{C}$ at $1.6^{\circ} \mathrm{C} / \mathrm{s}, 1 \mathrm{~min}$ at $65^{\circ} \mathrm{C}$ at $20^{\circ} \mathrm{C} / \mathrm{s}, 15 \mathrm{~s}$ at $95^{\circ} \mathrm{C}$ at $0.05^{\circ} \mathrm{C} / \mathrm{s}$.

\section{Behavioral Studies}

Map2k5 $5^{+/}$mutant mice and WT littermates were grouped by genotype and gender. Behavior studies were carried out from 9 to 25 weeks, and their body weights were tracked by an electronic weigher (NV621ZH, OHAUS, Parsippany NJ, United States) from 6 to 27 weeks.

\section{Rotarod}

Rotarod (ZH-600B, Anhui Zhenghua Co., Huaibei, China) was used to measure the motor coordination, learning ability, and fatigability of the mice. A rotarod device was placed in an SPF experiment room. Each mouse was placed in the room for $30 \mathrm{~min}$ for adaptation before running the experiments. Both WT and Map2 k5 $5^{+/-}$mutant mice aged 9 weeks were tested in the rotarod. During the learning period, both groups of mice were trained three times per day for continuous 3 days at a speed of $1 \mathrm{rpm}$ for $30 \mathrm{~s}$ and then at an accelerating speed from 1 to $10 \mathrm{rpm}$ by $1 \mathrm{rpm} / 30 \mathrm{~s}$ for $300 \mathrm{~s}$. On the day 4 , all mice were tested on the rotarod at an accelerating speed of from 4 to $30 \mathrm{rpm}$ for $500 \mathrm{~s}$ and 30 -min resting interval between each trial. The latency to fall off the rotarod was recorded.

\section{Open Field}

Open field device (XR-XZ301, Shanghai Xinruan Co., Shanghai, China) was placed in an SPF experiment room. It had infrared 
sensors, and the center was exposed to bright illumination (90$120 \mathrm{~lx}$ ). All experimental mice were aged 12 weeks. Each mouse was placed in the middle of one edge of the open field box and recorded for $20 \mathrm{~min}$. The activity of each mouse from 0 to $5 \mathrm{~min}$ was used for analysis. TopScan Lite Analysis Software (TopScan Lite, CleverSys) was used to collect and analyze data.

\section{Limbs' Strength}

Fore and four limbs strength were tested by the ability to hold on to a grip sensor (Bioseb G3, Chaville). Mice aged 13 weeks were tested. Each mouse was placed on a wire screen with their fore limbs or four limbs grasping to the grip. The grip sensor recorded the force of a metal grate being pulled in grams. We conducted three trials per mouse. The average strength of three trials was analyzed.

\section{Acoustic Startle Response (ASR) and Prepulse Inhibition (PPI)}

ASR and PPI apparatus Startle Response System (SR-LAB, San Diego, CA, United States) were used. We followed the standard protocols published by the International Mouse Phenotyping Consortium (IMPC) on the website ${ }^{2}$. Mice aged 17 weeks were tested. Each experiment was carried out at the late active phase from 7:00 to 8:00 a.m. The session was initiated with a 5min acclimation period (only background noise was used). In addition, it had an option to acclimate to the startle pulse in which $110-120 \mathrm{~dB} / 40-60 \mathrm{~ms}$ of white noise was presented alone, five times. These were excluded from the statistical analysis. Different prepulse trials of $20-\mathrm{ms}$ duration of white noise stimuli were presented alone $(\mathrm{PP} 1=72 \mathrm{~dB}, \mathrm{PP} 2=78 \mathrm{~dB}, \mathrm{PP} 3=84 \mathrm{~dB}$, or $\mathrm{PP} 4=90 \mathrm{~dB})$ and preceded the pulse by $70 \mathrm{~ms}(\mathrm{PP} 1+$ pulse, $\mathrm{PP} 2+$ pulse, $\mathrm{PP} 3+$ pulse, or PP4 + pulse) to derive the pre-pulse inhibition response. Startle pulse trials were started where $120 \mathrm{~dB} / 50 \mathrm{~ms}$ of white noise was presented alone. No stimulus trials in which only background noise was presented were used to measure baseline movement of the animal in the chamber. Background noise was $65-70 \mathrm{~dB}$. Startle responses were recorded and analyzed.

\section{Homecage Locomotor Activity}

Individual mice aged 25 weeks were placed in the Laboratory Animal Monitoring System (CLAMS, Columbus Instruments) for 24 -h adapting followed by 48 -h test. Data were collected and analyzed every $20 \mathrm{~min}$ in the system and resulted in 63 readings per individual a day. Total activity (TOT) and ambulatory activity (AMB) were measured based on the infrared beam breaks.

\section{Immunofluorescence Staining}

The cohorts included three female mice of each group aged postnatal 18 days (P18d), 21 weeks, 28 weeks, and 38 weeks. Mice were perfused with $4 \%$ paraformaldehyde (PFA) in phosphate-buffered saline (PBS) and sacrificed. Mouse brains were isolated on ice and fixed in 4\% PFA for at least $24 \mathrm{~h}$. Brain tissues were embedded with paraffin and serially cut into $5-\mu \mathrm{m}$-thick coronal sections. Brain sections of SNc and striatum were dewaxed by dimethylbenzene and gradient ethanol

\footnotetext{
${ }^{2}$ https://www.mousephenotype.org/impress/index
}

solutions. Citrate antigen retrieval solution was used, heated for $6 \mathrm{~min}$ at $65^{\circ} \mathrm{C}$, with 1 -min interval, and reheated for $6 \mathrm{~min}$ at $65^{\circ} \mathrm{C}$ for antigen retrieval. Every four continuous sections of $\mathrm{SNc}$ and striatum regions were stained in each mouse. The sections of mouse brains were immunostained at $4^{\circ} \mathrm{C}$ overnight with primary anti-tyrosine hydroxylase antibody (1:500, MAB318, Millipore Co., Massachusetts, United States), MAP2K5 (Concordet and Haeussler, 2018). Antibody (1:200, sc-135986, Santa Cruz Biotechnology, Inc., Northern California, United States), followed by Alexa Fluor 594conjugated secondary antibodies and 488-conjugated secondary antibodies (1:200, YEASEN Biotech Co., Ltd., Shanghai, China). Each section was incubated by 4',6-diamidino-2-phenylindole dihydrochloride (1:1,000, DAPI, Sigma-Aldrich, St. Louis, United States) for $20 \mathrm{~min}$ and treated with Antifade Mounting Medium (Beyotime Biotechnology, Shanghai, China). Images of each section were captured via fluorescence microscopy (Nikon Eclipse C1, Nikon, Tokyo, Japan). For each brain, three sections of substantia nigra pars compacta $(\mathrm{SNc})$ and striatum were analyzed. The $\mathrm{TH}^{+}$stained cell counts and mean immunofluorescence intensity per section were measured using Image (NIH).

\section{Western Blot}

Map2 $\mathrm{k}^{+/-}$mutant mice and WT littermates (female, aged 21 weeks, $n=4$ per group) were perfused with PBS and sacrificed. Brains were then dissected quickly within $10 \mathrm{~min}$ and snap frozen in liquid nitrogen. Proteins were prepared from the ventral midbrain (Yun et al., 2018) including nigrostriatal dopaminergic neurons. Cytoplasmic proteins were extracted by RIPA Lysis Buffer (Strong) (Beyotime Biotechnology, Shanghai, China) supplemented with Complete Protease Inhibitor Cocktail $(10 \times)$ and PhosSTOP ${ }^{\mathrm{TM}}$ Cocktail $(10 \times)$ (Roche Ltd., Basel, Switzerland). Western blots were performed using sodium dodecyl sulfate polyacrylamide gel electrophoresis and nitrocellulose membranes. Primary antibodies included anti-tyrosine hydroxylase $\mathrm{Ab}$ (1:1,000, MAB318, Millipore Co., Massachusetts, United States), Erk5 Antibody (1:1,000, \#3372, CST Ltd., Boston, United States), Phospho-Erk5 (1:1,000, Thr218/Tyr220), Antibody (1:1,000, \#3371, CST), MAP2K5 (Concordet and Haeussler, 2018). Antibody (1:200, sc-135986, Santa Cruz Biotechnology, Inc., Northern California, United States), and GAPDH (D16H11) XP ${ }^{\circledR}$ Rabbit mAb (1:1,000, \#5174, CST). Signals were detected with an ECL agent (Millipore). Blotting images were acquired and quantitatively analyzed by Image Lab Software (Bio-Rad, CA, United States).

\section{High-Performance Liquid Chromatography (HPLC)}

Striatal samples of 21-week-aged female mice were prepared for neurochemical measurements via HPLC analysis (Bionovogene Co., Ltd., Suzhou, China). The tissue was homogenized in $10 \%$ methanol formic solution-ddH2O (1:1) $(200 \mu)$ and centrifuged at a speed of $12,000 \mathrm{rpm}$ at $4^{\circ} \mathrm{C}$ for $5 \mathrm{~min}$. One hundred microliters of supernatant and $100 \mathrm{ppb}$ of double isotopes of L-valine (C13) L-phenylalanine (C13) internal standards (100 $\mu \mathrm{l})$ were mixed and passed through a $0.22-\mu \mathrm{m}$ filter membrane. 
Samples were injected in an ACQUITY UPLC ${ }^{\circledR}$ BEH C18 HPLC column $(2.1 \times 100 \mathrm{~mm}, 1.7 \mu \mathrm{m}$, Waters Co. $)$ at $40^{\circ} \mathrm{C}$. The mobile phase consisted of A-50\% methanol with $0.1 \%$ formic acid and B-10\% methanol with $0.1 \%$ formic acid. Compounds were measured with Analyst Software (AB SCIEX). The neurotransmitter metabolite peak areas were integrated and quantified against standard samples. The standard curves $r>0.99$, and the relative standard deviation (RSD) of the samples was $<9 \%$. Phenylalanine (Phe), tyrosine (Tyr), DOPA, dopamine (DA), epinephrine (Epi), glutamine (Gln), acetylcholine (ACh), serotonin, and 5-HIAA were measured. Results were normalized to the weight of each tissue and reported in $\mu \mathrm{g} / \mathrm{g}$.

\section{Statistics}

Analyses of mouse data were conducted via SPSS 26 (IBM). The test for genotype and gender-independent factor interactions of behavior studies were tested by two-way ANOVA followed by Tukey's post hoc tests for multiple comparisons in our behavior tests. Test for genotype effect of biochemical studies was conducted via Student's $t$-test, two-tailed analysis. A $p$-value of 0.05 was set as statistically significant.

\section{RESULTS}

\section{Characterization of Homozygous Map2k5-/- Mutant Embryos That Died at Mid-Gestation}

Map2k5 ${ }^{\text {em41 }}$ Cd71454 heterozygous Map2k5 $5^{+/-}$mice were viable and fertile. By tracking the body weight of Map2 $5^{+/-}$ and WT mice through 6-27 weeks, it was found that different genotypes exert no influence on body weight of mice, except for male mice at 11 weeks (Figure 1 in Supplementary Material 3).

However, Map2k5 $5^{\mathrm{em} 41} \mathrm{Cd} 71454$ homozygous Map2k5 $5^{-/-}$ mice died at mid-gestation with gross development retardation. At E9.5 (gestation day 9.5), homozygous Map2k5 $5^{-/-}$ mutant embryos showed normal phenotype. However, 9 out of $56(16.67 \%)$ homozygous Map2 $\mathrm{k} 5^{-/-}$embryos displayed developmental retardation at E10.5. Moreover, three homozygous Map2k5 $5^{-/-}$out 32 embryos at E11.5 (10.71\%) were found dead, 0/11 live embryos were found at E12.5, and 0/32 were found at E13.5. We deduced that Map2k5 ${ }^{\mathrm{em} 41} \mathrm{Cd} 71454$ strain's homozygous Map2 $\mathrm{k}^{-/-}$mutant embryos with exons 18-22 depletion died at approximately E11.5. This outcome was similar to the one found in a previous study reporting that Map2 $\mathrm{k}^{-/-}$mutant homozygous mice die at E10.5 due to a defect in fetal cardiogenesis (Wang et al., 2005). Thus, Map2 $\mathrm{k} 5^{+/-}$heterozygous mice and their WT littermates were used in the postnatal experiments.

\section{Map2k5+/- Heterozygous Mice Expressed Decreased Levels of Map2k5 mRNA in Different Organs}

To assess how effective the Map2k5 exons 18-20 gene deletion was on terminating transcription, the mRNA expression level of Map2 $\mathrm{k} 5^{+/-}$mutant mice was detected in different organs including the brain, heart, and spleen via qRT-PCR. We found a significant decreased expression levels of Map2k5 mRNA in all three different tissues (brain, heart, and spleen). Specifically, 20$30 \%$ relative quantitative levels of Map $2 \mathrm{k} 5 \mathrm{mRNA}$ were expressed in Map2 $\mathrm{k} 5^{+/-}$mutant mice compared with WT mice in the brain, heart, and spleen (referred to GAPDH) $(P=0.009,0.007$, 0.007, respectively, Figure 2A). Western blots of corresponding tissues were examined, which displayed a significant decrease in Map2k5 expression in the brain, and a less decrease in the heart and spleen (Figure 2B).

\section{Map2k5+/- Mice Displayed Sex-Dependent and Circadian-Related Motor Dysfunction}

Many movement disorders are circadian related. Based on the fact that RLS/WED is a disease usually presenting clinical signs in the evening and that women have higher risk than men ${ }^{1}$, we explored the profile of motor function in Map2k $5^{+/-}$mutant mice by performing a series of behavioral tests including open field test, rotarod test, and $12 \mathrm{~h}-12 \mathrm{~h}$ cycle infrared locomotor activity pattern. All these tests were done considering animal sex to assess for potential differences at this level. To exclude the
A

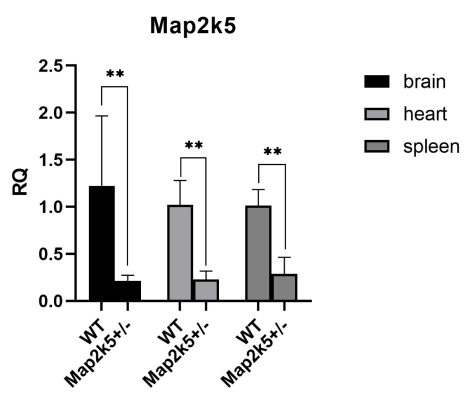

B

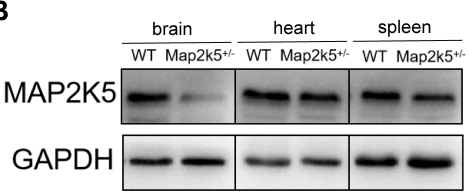

FIGURE 2 | (A) Map2k5 $5^{+/-}$heterozygous mice expressed 20-30\% relative quantitative (RQ) levels of intact Map2k5 mRNA in the brain, heart, and spleen ( $t$-test, $n=6)$. Bars represent means with standard errors of the mean (SEM). (B) Western blot of Map2k5 expressions in different tissues (brain, heart, and spleen) in WT and Map2k5+/- mice. ${ }^{* *} P<0.01$. 


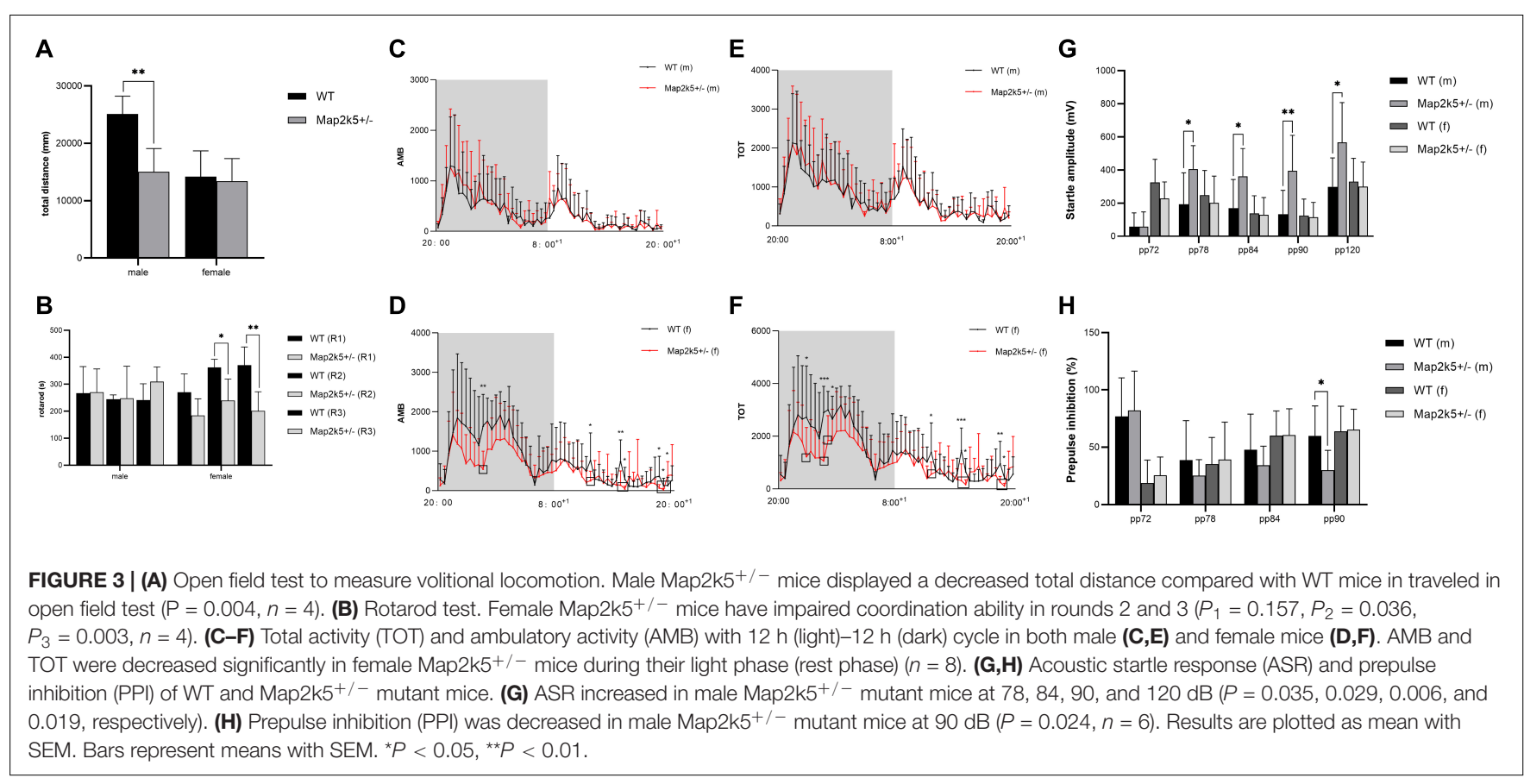

effect of muscle strengths in the motor test results, we examined the force of four and fore limbs in Map2 $\mathrm{k} 5^{+/-}$and WT mice.

Male Map2k5 $5^{+/-}$mice manifested a decreased total distance traveled in the open field test $(\mathrm{P}=0.004)$ when compared with WT mice, while female Map $2 \mathrm{k} 5^{+/-}$mice displayed no differences compared with the WT controls (Figure 3A). There was also a trend, but not a statistically significant difference, between different genotypes in which male Map2k5 $5^{+/-}$mice exhibited decreased central zone duration $(P=0.075$, figure not shown). These results indicated a different motor response to the new environment of Map2k5 mutant male mice. Previous studies showed that mice exploration behaviors could be influenced by hormones considering the open field cage as a new environment (Lalonde and Strazielle, 2017). Our results implied that male Map2k5+/- mice might display less motivation to explore and locomotion in the open field test.

As a measurement of motor coordination, learning, and fatigability, we evaluated the latency to fall from an accelerating rotarod three times on the test day following a 3-day learning period. It is important to consider that the three times repetition used in this test implies fatigue levels in each mouse. Female Map2k5 $5^{+/}$mutant mice displayed decreased latency to fall at the second and third rounds at the test day, and spared the first round of tests $\left(P_{1}=0.157, P_{2}=0.036, P_{3}=0.003\right.$, Figure 3B), suggesting increased fatigue propensity, impaired coordination and learning abilities, while male Map $2 \mathrm{k} 5^{+/-}$mice displayed no differences compared with WT mice. This indicated that Map2k5 deficiency in female mice may induce fatigue and poor coordination, similar to some motor symptoms presented in PD.

To analyze potential differences of volitional locomotion of Map2 $\mathrm{k} 5^{+/-}$mutant mice subjected to a circadian variation, we collected motor data per $20 \mathrm{~min}$ in a cage equipped with infrared beams (divided a whole day into 63 equal periods).
Total activity (TOT, which includes when animals scratches themselves and move their limbs without locomotion) and ambulatory activity (AMB, which only includes their body movement) were recorded with a $12 \mathrm{~h}$ (light)-12 h (dark) cycle for a day. Through this method, we found that there was interaction between genders and genotypes $(p=0.043)$; the main effect of genotype on both AMB and TOT in female mice was significant. Specifically, AMB and TOT of female Map2 $\mathrm{k} 5^{+/-}$ mutant mice decreased significantly during their sleeping time (light-on phase, Figures 3D,F), implying a hypokinetic and bradykinetic phenotype (Kim et al., 2016; Langley et al., 2017). However, male Map2k5 $5^{+/}$mutant mice did not display any differences compared with their WT littermates (Figures 3C,E). This provided us with a gender-dependent, circadian-related movement dysfunction profile, contrary to RLS/WED symptoms to some extent (Allen et al., 2014).

When considering the gender-dependent different results of $12 \mathrm{~h}-12 \mathrm{~h}$ locomotor activity trace [significant decreased sleeping body movements (AMB and TOT) only in female mice] and open field tests (only male mice showed a significant reduction in total distance in open field), we thought that as a novel environment exposure, mice were also experienced in the open filed apparatus, the behavior found in male mice indicated stress-based movement in these mice (Lalonde and Strazielle, 2017; Zhang et al., 2019), whereas $12 \mathrm{~h}-12 \mathrm{~h}$ locomotor activity trace data were more similar to the nature pattern of movement, including circadian-distinctive manners. Therefore, male Map2k5-deficient mice motor dysfunction can be interpreted into a stress-based alteration, while female Map2k5 deficient mice movement phenotypes are basal changes due to motor-control pathway dysfunctions.

To discard the effect of limbs' strength on the behavioral performance, each mouse's fore and four limbs' strengths were 

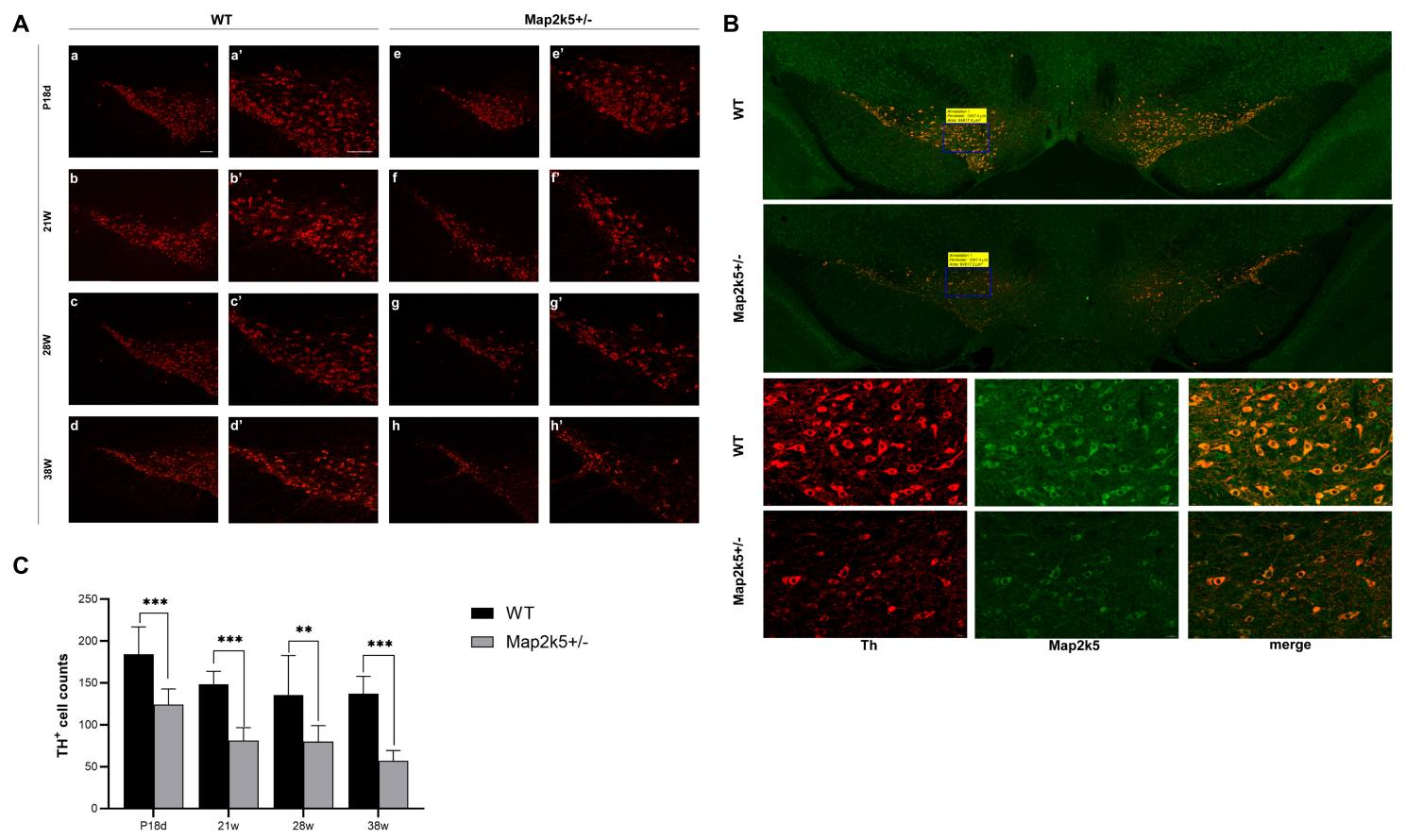

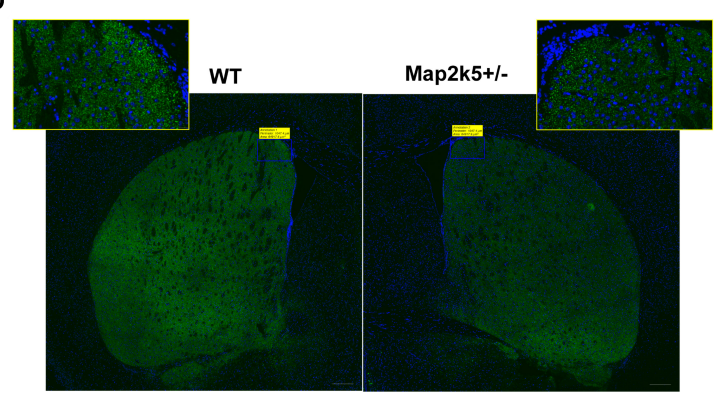

E

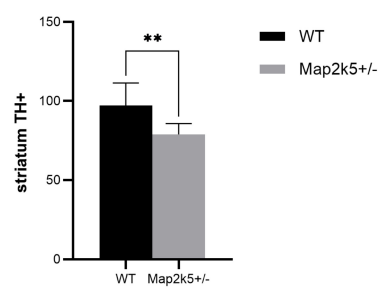

FIGURE 4 | (A) Coronal sections of substantia nigra pars compacta (SNc) were prepared from P18d, 21, 28, and 38 weeks and immunostained with an anti-TH antibody. Map2k5 $5^{+/-}$mutant mice had decreased $\mathrm{TH}^{+}$cell number in SNc at P18d, 21, 28, and 32 weeks. (a-d) Immunostained TH ${ }^{+}$cells in SNc of Map2k5+/+ WT mice (100x). (e-h) TH+ cells in SNc of Map2k5+/- mutant mice (100x). (a'-h') High-magnification images (200 x ) of the corresponding section in (a-h). Scale bar, $100 \mu \mathrm{m}$. (B) $\mathrm{TH}^{+}$and Map2k5+-stained cells, and merged pictures of Map2k5+/- mutant and WT mice in SNc of at 38 weeks. Low-magnification images $(50 \times)$ and high-magnification images $(400 \times) .200 \mu \mathrm{m}(50 \times), 20 \mu \mathrm{m}(400 \times)$. (C) TH+ cell counts in SNc were reduced in female Map2k5+/- mutant mice compared with WT mice at P18d, 21, 28, and 38 weeks $\left(P_{18 d}<0.001, P_{21 w}<0.001, P_{28 w}=0.005, P_{38 w}<0.001\right)$. (D) Coronal sections of striatum immunostained with anti-TH antibody. We found decrease immunofluorescence in Map2k5 $5^{+/}$mutant mice compared with WT mice. Low-magnification images (50 $\times$) and high-magnification images $(630 \times)$. Scale bar, $200 \mu \mathrm{m}(50 \times), 20 \mu \mathrm{m}(630 \times)$. (E) Mean immunofluorescence intensity of each slice was analyzed. There was decreased TH density in Map2k5 ${ }^{+/-}$mutant mice $(P=0.006)$. Results are plotted as mean with SEM. Bars represent means with SEM. ${ }^{\star \star} P<0.01$, ${ }^{\star \star \star} P<0.001$.

tested. The results showed that the limbs' force has no difference between WT and Map2 $\mathrm{k} 5^{+/-}$mutant mice in both male and female subjects (Figure 2 in Supplementary Material 3).

\section{Male Map2k5+/- Mutant Mice Display Sensorimotor Gating Deficits}

The main complaint of+oldd unpleasant feelings displayed by RLS/WED patients is hard to evaluate in mice. In order to measure this phenotype, we tested the sensorimotor gating levels on each group of mice. The sensory response adaptation was recorded by acoustic startle response (ASR) and prepulse inhibition (PPI) tests. Male Map2k5 $5^{+/-}$mutant mice represented a decreased ASR at 78, 84, 90, and $120 \mathrm{~dB}$ ( $P=0.035,0.029,0.006$, and 0.019, respectively, Figure 3G), and PPI results implied sensorimotor gating deficits at $90 \mathrm{~dB}$ $(p=0.024$, Figure $3 \mathbf{H})$. This implied that male Map2k $5^{+/-}$ mutant mice displayed deficits on sensory stimulus, but not female mutant mice. ASR and PPI tests examined the intact function of the vestibular system, auditory system, ventrolateral tegmental nucleus, pontine reticular formation, and their interactions (Yeomans et al., 2002). In addition, there is also evidence showing that the amygdala, accumbens nuclei, and other limbic systems contribute to the performance of ASR 


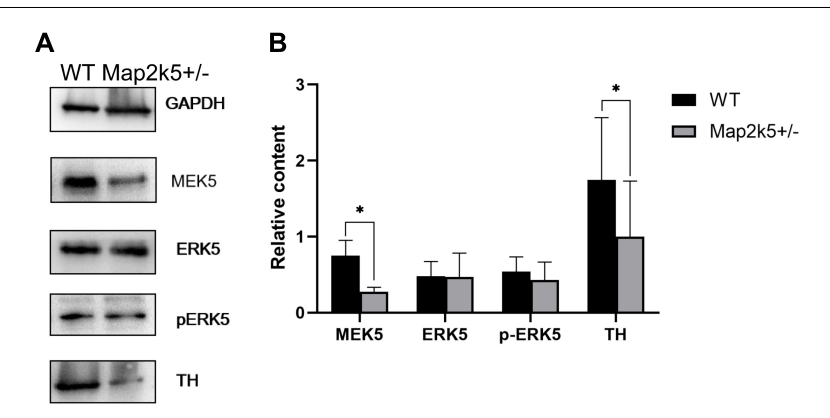

FIGURE 5 | (A) Representative Western blots images of MAP2K5, ERK5, phosphorylated ERK5 (active), TH protein, and GAPDH are depicted. (B) Data from male WT and Map2k5+/- mutant mice were pooled for this analysis. Bar graph is hemi-quantitative analysis of the Western blot, showing a significant decrease in MAP2K5 and TH $\left(\mathrm{P}_{\text {Map2k5 }}=0.023, \mathrm{P}_{\mathrm{TH}}=0.013\right.$, respectively) in the Map2 $\mathrm{k} 5^{+/-}$mutant mice. Results are plotted as mean with SEM (paired $t$-test, $n=4$ ). Results are plotted as mean with SEM. Bars represent means with SEM. ${ }^{\star} P<0.05$.

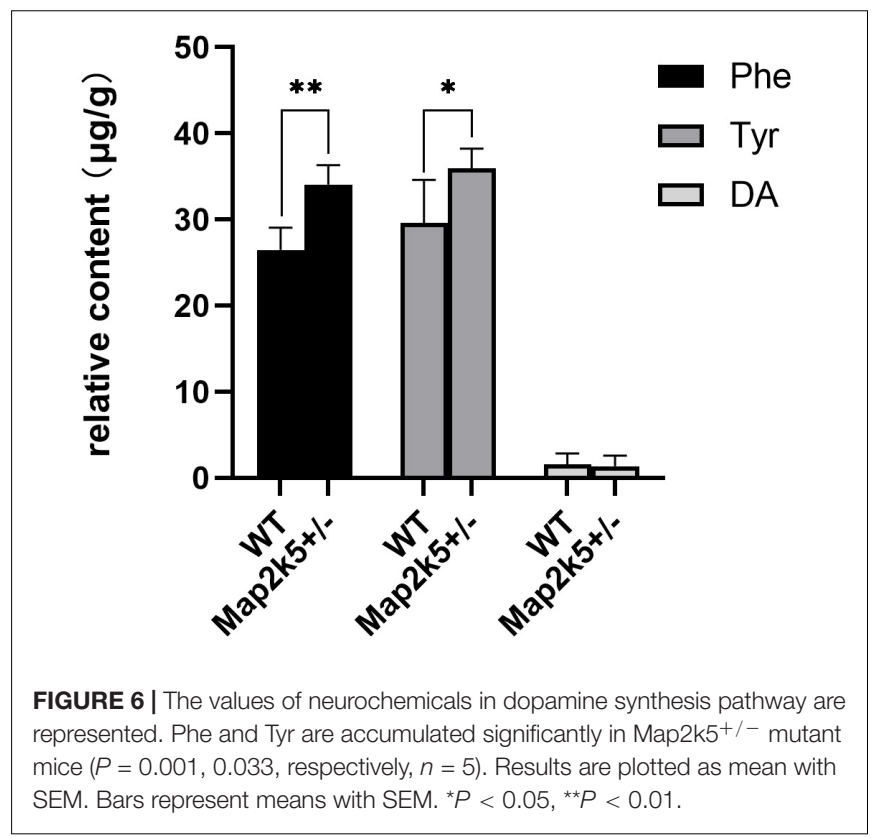

and PPI (Frau et al., 2019). Considering the results we got from open field and PPI, where only male mutant mice displayed stress-related sensorimotor deficits, and taking in account that both tests involve the accumbens nucleus, amygdala nucleus, and mesolimbic dopamine system (that participate in many psychiatric disorders, such as schizophrenia, depression, obsessive-compulsive disorder, psychological substance-induced behavior, and so on), we hypothesized that sex hormone levels might affect the behaviors of Map2 $\mathrm{k} 5^{+/-}$mutant mice where male mice was prone to manifest stress-related responses (Pittenger et al., 2016; Khan and Powell, 2018; Liu et al., 2018; Bilel et al., 2019; Tapias-Espinosa et al., 2019).

\section{The Quantity of SNc and Striatum $\mathrm{TH}^{+}$ Cells Were Reduced in Map2k5+/- Mutant Mice}

Based on the behavior tests' results found in female mice, we thought that the phenotype of bradykinesia, poor coordination, and fatigue was similar to the motor dysfunction caused by nigrostriatal pathway deficits. Dopaminergic neurons are crucial for the nigrostriatal pathway, so we assessed the levels of dopaminergic neurons by immunostaining of $\mathrm{TH}$ protein (the rate-limiting enzyme in the dopamine synthesis pathway) in SNc. We assessed the $\mathrm{TH}^{+}$cell counts in the $\mathrm{SNc}$ region of the mice brain. $\mathrm{TH}^{+}$cells were stained and captured by florescence microscopy (Figure 4A), and a montaged image of Map2k5stained section was captured, which indicated a synchronous declination of Map2k5 and TH protein (Figure 4B). Results showed a decreased $\mathrm{SNc} \mathrm{TH}^{+}$cell number in female Map2 $\mathrm{k}^{+/-}$ mutant mice at P18d, 21 weeks, 28 weeks, and 38 weeks compared with WT mice $\left(\mathrm{P}_{18 \mathrm{~d}}<0.001, \mathrm{P}_{21 \mathrm{w}}<0.001, \mathrm{P}_{28 \mathrm{w}}=0.005\right.$, $\mathrm{P}_{38 \mathrm{w}}<0.001$, Figure $4 \mathrm{C}$ ). The reduction of $\mathrm{TH}^{+}$cells in $\mathrm{SNc}$ suggested that MAP2K5 might be involved in the survival of dopaminergic cells in SNc early in life. At $\mathrm{P} 18 \mathrm{~d}$, the $\mathrm{TH}^{+}$cells of Map2k5 $5^{+/-}$mutant mice were $70 \%$ of their WT littermates, $50-$ $60 \%$ at 21 and 28 weeks, while this number declined to about $40 \%$ at age by 38 weeks. Thus, Map2k $5^{+/-}$deficiency might disturb the dopamine system in $\mathrm{SNc}$, and the effect of Map2 $\mathrm{k}^{+/-}$ deficiency on losing dopaminergic neurons increased with aging.

$\mathrm{SNc}$ dopaminergic neurons project to the striatum forming the nigrostriatal pathway and facilitate movement via direct and indirect pathways. We conducted immunostaining of $\mathrm{TH}+$ protein in the striatum of 21-week female mice to evaluate the dopaminergic neural fibers density in this area, where DA is released and acts on the postsynaptic membrane. Immunofluorescence images of the striatum displayed a reduction in TH intensity (Figure 4D). Mean immunofluorescence intensity was calculated for each tissue slice, showing a significant decrease in dopaminergic neuron fibers' density in female Map2 $5^{+/-}$mutant mice compared with WT mice ( $p=0.006$, Figure $4 \mathrm{E}$ ). These results confirmed the decreased dopaminergic neurons in Map2 $\mathrm{k}^{+/-}$mutant mice, consistent with the movement phenotypes displayed in behavior tests.

\section{Decreased Quantities of Map2k5 and TH Protein, but Not pErk5 in Map2k5 $5^{+/-}$ Mutant Mice}

To explore the mechanism of reduced dopaminergic neurons in Map2 $\mathrm{k}^{+/-}$mutant mice, we measured the level of Map2k5Erk5 pathway molecules and TH protein in ventral midbrain including dopaminergic neurons in the nigrostriatal pathway. Four female mice aged 21 weeks for each genotype were used. Proteins of MAP2K5 (MEK5), ERK5, phosphorylated ERK5 (active), TH, and GAPDH were stained (Figure $\mathbf{5 A}$ ). The ratio of each protein was calculated by its relative quantity referred to the GAPDH band. The results implied a synchronized decrease in Map2k5 and TH proteins in the ventral midbrain 
TABLE 1 | Levels of phenylalanine (Phe), tyrosine (Tyr), dopamine (DA), epinephrine (Epi), serotonin, and its metabolite 5-HIAA in the striatum.

\begin{tabular}{lccc}
\hline Neurochemicals & WT & Map2k5+/- & P-value \\
\hline Phe & $26.46 \pm 1.17$ & $34.04 \pm 1.01$ & $0.001^{\text {** }}$ \\
Tyr & $29.62 \pm 2.24$ & $35.95 \pm 1.01$ & $0.033^{*}$ \\
DA & $1.55 \pm 0.59$ & $1.34 \pm 0.63$ & 0.815 \\
Epi & $1.50 \pm 0.62$ & $1.07 \pm 0.46$ & 0.621 \\
Gln & $675.43 \pm 189.03$ & $574.01 \pm 163.44$ & 0.695 \\
ACh & $0.58 \pm 0.17$ & $0.22 \pm 0.07$ & 0.094 \\
Serotonin & $0.04 \pm 0.00$ & $0.03 \pm 0.02$ & 0.743 \\
5-HIAA & $0.35 \pm 0.11$ & $0.26 \pm 0.05$ & 0.478 \\
\hline
\end{tabular}

The values of neurochemicals are represented in means $\pm S E M(\mu \mathrm{g} / \mathrm{g})$ of the tissue (t-test, $n=5$ ). ${ }^{*} P<0.05$, ${ }^{* *} P<0.01$.

$\left(\mathrm{P}_{\text {Map } 2 \mathrm{k} 5}=0.023, \mathrm{P}_{\mathrm{TH}}=0.013\right.$, respectively $)$, while Erk5 was not reduced, and phosphorylated Erk5 was reduced without a statistical significance $(P=0.324$, Figure $5 \mathrm{~B})$. It is possible that MAP2K5 deficiency decreases TH expression in an ERK5independent manner. However, more studies are needed to clarify the molecular mechanism of this phenomenon.

\section{Dopamine Levels Were Not Altered in Striatum, but Its Substrates Were Accumulated}

As TH is the key enzyme of dopamine synthesis and Map2k5deficient mice showed dopaminergic neuron deficiency in the nigrostriatal pathway, we tried to investigate whether Map2k5 deficiency-induced TH reduction had an effect on neurotransmitters and their metabolites (Obara et al., 2016). We used HPLC to examine monoamine neurochemical systems in the striatum on five female 21-week-old mice per group. The substrates of DA in its synthesis pathway (Phe and Tyr) were accumulated significantly $(P=0.001,0.033$, respectively, Figure 6) in Map2 $\mathrm{k} 5^{+/}-$female mutant mice compared with WT female mice, while the gross DA content decreased but without significant difference between WT and Map2k5 $5^{+/-}$mutant mice. Other neurotransmitters, such as Gln, ACh, serotonin, and its metabolite 5-HIAA were not altered in Map2 $\mathrm{k} 5^{+/-}$mutant mice (Table 1). The accumulation of substrates of phenylalanine and tyrosine is consistent with the result of decreased $\mathrm{TH}$ enzyme in the nigrostriatal pathway. However, there was no statistical difference on the dopamine levels between Map2 $\mathrm{k} 5^{+/-}$mutant and WT mice. This inconsistent finding may be due to two reasons. First, the dopamine level in the striatum was very low to be detected, beyond the linear dynamic range of the HPLC standard curve. This problem may be solved by other techniques like fluorometric detection (Ghani et al., 2021). Second, TH reduction in Map2k5-deficient mouse model was not strong enough to create a significant reduction in dopamine in the nigrostriatal pathway in Map2k5 mutant mice. Interestingly, previous studies showed that DA was not significantly reduced when $\mathrm{TH}$ protein level decreased significantly at 3 weeks in Th mutant mice. However, at 1 year old, when $\mathrm{TH}$ content reduced at $3 \%$ of WT mice, DA reduced to a significant level $(P<0.001)$ (Korner et al., 2015). Therefore, there may be a threshold of $\mathrm{TH}$ reduction when DA decreased into a significant level.

\section{DISCUSSION}

In this study, we described for the first time the movement phenotypes and underlying dopaminergic disturbance in a Map2k5-deficient mouse model. These behavioral changes were contrary to RLS/WED symptoms, but similar to PD in some aspects, including reduced circadian-dependent ambulatory locomotion, coordination, and balance ability. Similarly, reduced dopaminergic neurons and $\mathrm{TH}$ protein in the nigrostriatal pathway resembled some pathological aspects of PD. Actually, both diseases share many common features, such as high prevalence of comorbidity of RLS/WED with PD, dopaminergic dysregulation in the pathogenesis of both diseases, and good response to dopamine agonists (Splinter, 2007). This suggests similar mechanisms that might be shared by both disorders (Alonso-Navarro et al., 2019). In addition, SNPs of MAP2K5 have been shown to be associated with the reduced risk of RLS/WED (Winkelmann et al., 2007; Yang et al., 2011; Moore et al., 2014; Li et al., 2017), but increased the risk of tremor in $\mathrm{PD}$ ( $\mathrm{rs} 12593813,61.0 \%$ vs. $46.5 \%, p=0.001$ ) (Gan-Or et al., 2015). This further indicates a close relationship, but probably opposite, effect of MAP2K5 in RLS/WED and PD. Also, PD patients usually present with odor problems, and previous studies reported deficits in odor discrimination in the conditional deletion of Erk5 mouse (Zou et al., 2012). Therefore, we hypothesized that the Map2k5-Erk5 pathway might be involved in the pathophysiology of both RLS/WED and PD.

Several in vitro studies found that the MAP2K5-ERK5 pathway was crucial in dopaminergic neuron survival. It protected dopaminergic neurons under both basal conditions and in response to oxidative stress (Cavanaugh et al., 2006). MAP2K5-ERK5 was also involved in Mn-induced cytotoxicity, altering neurons' survival and DA production in MN9D cells (Ding et al., 2020). ERK5 was reported to induce ankrd1 for catecholamines biosynthesis and homeostasis in adrenal gland medullary cells (Obara et al., 2016). Thus, it is reasonable to hypothesize that reduced dopaminergic neurons and $\mathrm{TH}$ protein in our Map2k5-deficient mouse model might be due to decreased Map2k5-Erk5 pathway. However, p-Erk5 level remained unchanged in our Map2k5-deficient mouse. Based on the fact that the level of p-ERK5 dropped greatly after birth (Parmar et al., 2015) and Erk5 is the only present known substrate of Map2k5, the regulation of the Map2k5-Erk5 pathway on dopaminergic neuron probably occurred in the early developmental stage. Actually, Map2k5-Erk5 seems to be involved in protecting embryonic, but not mature, cortical neurons in an in vitro study (Liu et al., 2003, 2006). Also, conditional deletion of Erk5 in the nervous system was once reported for impaired neuron migration into the olfactory bulb during nervous system development at prenatal stages (Li et al., 2013). Since the heterozygous Map2k5 mutant mice already displayed reduction in $\mathrm{TH}^{+}$dopaminergic cells significantly on postnatal 18 days in our study, it is plausible to think that the Map2k5-Erk5 pathway regulates the dopaminergic development in an earlier period and contributes to central nervous system disorders such as RLS/WED and PD. Actually, we aim to explore the Map2k5-deficient 
effect on dopaminergic neuron development of early neurogenesis in our future research.

Additionally, our results of behavioral tests displayed sexual distinctions. Here, we uncovered the nigrostriatal dopaminergic system disturbance in female Map2k5-deficient mice, while the underlying mechanism of stress-related alterations in sensorimotor response of male Map2k5-deficient mice, involving amygdala, hippocampus, and accumbens nuclei in the limbic system (Lalonde and Strazielle, 2017; Frau et al., 2019) is worth to be further studied.

There are limitations of our study. The dopamine level in the striatum was very low to be detected. As a matter of fact, several heterozygous samples' DA concentrations were beyond the linear dynamic range of the HPLC standard curve. It could indeed account to difficulties to detect any difference in DA between groups. This problem may be solved by other techniques like fluorometric detection (Ghani et al., 2021). What is more, our results showed significant accumulation of substrates (Phe and Tyr) of dopamine before the step mediated by TH accompanied by insignificant reduction in DA, so we hypothesized that a threshold may exist where the reduction in $\mathrm{TH}$ induces $\mathrm{DA}$ decrease into a significant level.

\section{CONCLUSION}

In conclusion, Map2k5-deficient mice manifest movement impairments, consistent with reductions in dopaminergic neurons and $\mathrm{TH}$ protein in the nigrostriatal dopaminergic system. These features confirmed that dopaminergic disturbance by the Map2k5-Erk5 pathway was involved in the pathogenesis of movement disorders like RLS/WED and PD. In addition, our Map2k5-deficient mouse model provides a unique tool to further investigate the role of the Map2k5-Erk5 pathway in other central nervous system disorders with dopamine deficits, such as schizophrenia.

\section{DATA AVAILABILITY STATEMENT}

The raw data supporting the conclusions of this article will be made available by the authors, without undue reservation.

\section{REFERENCES}

Accili, D., Fishburn, C. S., Drago, J., Steiner, H., Lachowicz, J. E., Park, B. H., et al. (1996). A targeted mutation of the D3 dopamine receptor gene is associated with hyperactivity in mice. Proc. Natl. Acad. Sci. U.S.A. 93, 1945-1949. doi: 10.1073/pnas.93.5.1945

Allen, R. P., Picchietti, D. L., Garcia-Borreguero, D., Ondo, W. G., Walters, A. S., Winkelman, J. W., et al. (2014). Restless legs syndrome/Willis-Ekbom disease diagnostic criteria: updated International Restless Legs Syndrome Study Group (IRLSSG) consensus criteria-history, rationale, description, and significance. Sleep Med. 15, 860-873. doi: 10.1016/j.sleep.2014.03.025

Alonso-Navarro, H., García-Martín, E., Agúndez, J. A. G., and Jiménez-Jiménez, F. J. (2019). Association between restless legs syndrome and other movement disorders. Neurology 92, 948-964. doi: 10.1212/wnl.0000000000007500

\section{ETHICS STATEMENT}

The animal study was reviewed and approved by the Ruijin Hospital, Shanghai Jiao Tong University School of Medicine.

\section{AUTHOR CONTRIBUTIONS}

YH contributed to the project configuration, experiment investigation, data analysis, and original manuscript description. JM contributed to the project supervision, data analysis manuscript modification, and funding acquisition. RM contributed to the manuscript modification and data curation. PW and QL participated in the experiment investigation, data analysis, and methodology support. All authors contributed to the article and approved the submitted version.

\section{FUNDING}

This work was supported by the National Natural Science Foundation of China (81571103) and the National Key R\&D Program of China (2016YFC1306000).

\section{ACKNOWLEDGMENTS}

We thank Minghan Tong (Shanghai Institute of Biochemistry and Cell Biology, Chinese Academy of Sciences) for technical assistance and manuscript modifications. We thank GemPharmmatech Co., Ltd. for technical assistance and cooperation.

\section{SUPPLEMENTARY MATERIAL}

The Supplementary Material for this article can be found online at: https://www.frontiersin.org/articles/10.3389/fnagi. 2021.651638/full\#supplementary-material

Appari, M., Breitbart, A., Brandes, F., Szaroszyk, M., Froese, N., Korf-Klingebiel, M., et al. (2017). C1q-TNF-Related Protein-9 promotes cardiac hypertrophy and failure. Circ. Res. 120, 66-77. doi: 10.1161/circresaha.116.309398

Bilel, S., Tirri, M., Arfè, R., Stopponi, S., Soverchia, L., Ciccocioppo, R., et al. (2019). Pharmacological and behavioral effects of the synthetic cannabinoid AKB48 in Rats. Front. Neurosci. 13:1163. doi: 10.3389/fnins.2019. 01163

Cabrera-Mendoza, B., Martínez-Magaña, J. J., Genis-Mendoza, A. D., Sarmiento, E., Ruíz-Ramos, D., Tovilla-Zárate, C. A., et al. (2020). High polygenic burden is associated with blood DNA methylation changes in individuals with suicidal behavior. J. Psychiatr. Res. 123, 62-71. doi: 10.1016/j.jpsychires.2020. 01.008

Castro, M., Laviña, B., Ando, K., Álvarez-Aznar, A., Abu Taha, A., Brakebusch, C., et al. (2019). CDC42 deletion elicits cerebral vascular malformations via 
increased MEKK3-dependent KLF4 expression. Circ. Res. 124, 1240-1252. doi: $10.1161 /$ circresaha. 118.314300

Cavanaugh, J. E., Jaumotte, J. D., Lakoski, J. M., and Zigmond, M. J. (2006). Neuroprotective role of ERK1/2 and ERK5 in a dopaminergic cell line under basal conditions and in response to oxidative stress. J. Neurosci. Res. 84, 1367-1375. doi: 10.1002/jnr.21024

Concordet, J. P., and Haeussler, M. (2018). CRISPOR: intuitive guide selection for CRISPR/Cas9 genome editing experiments and screens. Nucleic Acids Res. 46(W1), W242-W245. doi: 10.1093/nar/gky354

Dinev, D., Jordan, B. W., Neufeld, B., Lee, J. D., Lindemann, D., Rapp, U. R., et al. (2001). Extracellular signal regulated kinase 5 (ERK5) is required for the differentiation of muscle cells. EMBO Rep. 2, 829-834. doi: 10.1093/emboreports/kve177

Ding, H., Wang, F., Su, L., Zhao, L., Hu, B., Zheng, W., et al. (2020). Involvement of MEK5/ERK5 signaling pathway in manganese-induced cell injury in dopaminergic MN9D cells. J. Trace Elem. Med. Biol. 61:126546. doi: 10.1016/ j.jtemb.2020.126546

English, J. M., Pearson, G., Baer, R., and Cobb, M. H. (1998). Identification of substrates and regulators of the mitogen-activated protein kinase ERK5 using chimeric protein kinases. J. Biol. Chem. 273, 3854-3860. doi: 10.1074/jbc.273.7. 3854

Frau, R., Miczán, V., Traccis, F., Aroni, S., Pongor, C. I., Saba, P., et al. (2019). Prenatal THC exposure produces a hyperdopaminergic phenotype rescued by pregnenolone. Nat. Neurosci. 22, 1975-1985. doi: 10.1038/s41593-0190512-2

Gan-Or, Z., Alcalay, R. N., Bar-Shira, A., Leblond, C. S., Postuma, R. B., BenShachar, S., et al. (2015). Genetic markers of restless legs syndrome in Parkinson disease. Parkinsonism Relat. Disord. 21, 582-585. doi: 10.1016/j.parkreldis.2015. 03.010

Ghani, S. M., Rezaei, B., Jamei, H. R., and Ensafi, A. A. (2021). Novel synthesis of a dual fluorimetric sensor for the simultaneous analysis of levodopa and pyridoxine. Anal. Bioanal. Chem. 413, 377-387. doi: 10.1007/s00216-02003005-9

Jo, M., Chung, A. Y., Yachie, N., Seo, M., Jeon, H., Nam, Y., et al. (2017). Yeast genetic interaction screen of human genes associated with amyotrophic lateral sclerosis: identification of MAP2K5 kinase as a potential drug target. Genome Res. 27, 1487-1500. doi: 10.1101/gr.211649.116

Johnson, G. L., and Lapadat, R. (2002). Mitogen-activated protein kinase pathways mediated by ERK, JNK, and p38 protein kinases. Science 298, 1911-1912. doi: 10.1126/science.1072682

Kang, S. G., Lee, Y. J., Park, Y. M., Kim, L., and Lee, H. J. (2018). Haplotype association of the MAP2K5 gene with antipsychotics-induced symptoms of restless legs syndrome among patients with schizophrenia. Psychiatry Investig. 15, 84-89. doi: 10.4306/pi.2018.15.1.84

Kato, Y., Zhao, M., Morikawa, A., Sugiyama, T., Chakravortty, D., Koide, N., et al. (2000). Big mitogen-activated kinase regulates multiple members of the MEF2 protein family. J. Biol. Chem. 275, 18534-18540. doi: 10.1074/jbc.M0015 73200

Khan, A., and Powell, S. B. (2018). Sensorimotor gating deficits in "two-hit" models of schizophrenia risk factors. Schizophr. Res. 198, 68-83. doi: 10.1016/j.schres. 2017.10.009

Kim, B. W., Jeong, K. H., Kim, J. H., Jin, M., Kim, J. H., Lee, M. G., et al. (2016). Pathogenic upregulation of glial lipocalin-2 in the Parkinsonian dopaminergic system. J. Neurosci. 36, 5608-5622. doi: 10.1523/jneurosci.4261-15.2016

Korner, G., Noain, D., Ying, M., Hole, M., Flydal, M. I., Scherer, T., et al. (2015). Brain catecholamine depletion and motor impairment in a Th knock-in mouse with type B tyrosine hydroxylase deficiency. Brain 138(Pt 10), 2948-2963. doi: 10.1093/brain/awv224

Lalonde, R., and Strazielle, C. (2017). Neuroanatomical pathways underlying the effects of hypothalamo-hypophysial-adrenal hormones on exploratory activity. Rev. Neurosci. 28, 617-648. doi: 10.1515/revneuro-2016-0075

Langley, M., Ghosh, A., Charli, A., Sarkar, S., Ay, M., Luo, J., et al. (2017). MitoApocynin prevents mitochondrial dysfunction, microglial activation, oxidative damage, and progressive Neurodegeneration in MitoPark transgenic mice. Antioxid Redox Signal. 27, 1048-1066. doi: 10.1089/ars.2016.6905

Li, G., Tang, H., Wang, C., Qi, X., Chen, J., Chen, S., et al. (2017). Association of BTBD9 and MAP2K5/SKOR1 with restless legs syndrome in Chinese population. Sleep 40:zsx028. doi: 10.1093/sleep/zsx028
Li, T., Pan, Y. W., Wang, W., Abel, G., Zou, J., Xu, L., et al. (2013). Targeted deletion of the ERK5 MAP kinase impairs neuronal differentiation, migration, and survival during adult neurogenesis in the olfactory bulb. PLoS One 8:e61948. doi: 10.1371/journal.pone.0061948

Liu, F., Zhang, H., and Song, H. (2017). Upregulation of MEK5 by Stat3 promotes breast cancer cell invasion and metastasis. Oncol. Rep. 37, 83-90. doi: 10.3892/ or.2016.5256

Liu, L., Cavanaugh, J. E., Wang, Y., Sakagami, H., Mao, Z., and Xia, Z. (2003). ERK5 activation of MEF2-mediated gene expression plays a critical role in BDNF-promoted survival of developing but not mature cortical neurons. Proc. Natl. Acad. Sci. U.S.A. 100, 8532-8537. doi: 10.1073/pnas.13328 04100

Liu, L., Cundiff, P., Abel, G., Wang, Y., Faigle, R., Sakagami, H., et al. (2006). Extracellular signal-regulated kinase (ERK) 5 is necessary and sufficient to specify cortical neuronal fate. Proc. Natl. Acad. Sci. U.S.A. 103, 9697-9702. doi: 10.1073/pnas.0603373103

Liu, Y. P., Yang, Y. Y., Wan, F. J., and Tung, C. S. (2018). Importance of intervention timing in the effectiveness of antipsychotics. Prog. Neuropsychopharmacol. Biol. Psychiatry 81, 493-500. doi: 10.1016/j.pnpbp. 2017.02.003

Moore, Ht, Winkelmann, J., Lin, L., Finn, L., Peppard, P., and Mignot, E. (2014). Periodic leg movements during sleep are associated with polymorphisms in BTBD9, TOX3/BC034767, MEIS1, MAP2K5/SKOR1, and PTPRD. Sleep 37, 1535-1542. doi: 10.5665/sleep.4006

Obara, Y., Nagasawa, R., Nemoto, W., Pellegrino, M. J., Takahashi, M., Habecker, B. A., et al. (2016). ERK5 induces ankrd1 for catecholamine biosynthesis and homeostasis in adrenal medullary cells. Cell Signal. 28, 177-189. doi: 10.1016/j. cellsig.2015.12.016

Parmar, M. S., Jaumotte, J. D., Zigmond, M. J., and Cavanaugh, J. E. (2015). ERK1, 2, and 5 expression and activation in dopaminergic brain regions during postnatal development. Int. J. Dev. Neurosci. 46, 44-50. doi: 10.1016/j.ijdevneu. 2015.06.009

Pittenger, C., Adams, T. G. Jr., Gallezot, J. D., Crowley, M. J., Nabulsi, N., James, R., et al. (2016). OCD is associated with an altered association between sensorimotor gating and cortical and subcortical 5-HT1b receptor binding. J. Affect. Disord. 196, 87-96. doi: 10.1016/j.jad.2016. 02.021

Simões, A. E., Pereira, D. M., Gomes, S. E., Brito, H., Carvalho, T., French, A., et al (2015). Aberrant MEK5/ERK5 signalling contributes to human colon cancer progression via NF-кB activation. Cell Death Dis. 6:e1718. doi: 10.1038/cddis. 2015.83

Splinter, M. Y. (2007). Rotigotine: transdermal dopamine agonist treatment of Parkinson's disease and restless legs syndrome. Ann. Pharmacother. 41, 285295. doi: 10.1345/aph.1H113

Tapias-Espinosa, C., Río-Álamos, C., Sánchez-González, A., Oliveras, I., Sampedro-Viana, D., Castillo-Ruiz, M. D. M., et al. (2019). Schizophrenialike reduced sensorimotor gating in intact inbred and outbred rats is associated with decreased medial prefrontal cortex activity and volume. Neuropsychopharmacology 44, 1975-1984. doi: 10.1038/s41386-0190392-X

Wang, X., Merritt, A. J., Seyfried, J., Guo, C., Papadakis, E. S., Finegan, K. G., et al. (2005). Targeted deletion of mek5 causes early embryonic death and defects in the extracellular signal-regulated kinase 5/myocyte enhancer factor 2 cell survival pathway. Mol. Cell Biol. 25, 336-345. doi: 10.1128/mcb.25.1.336-345. 2005

Winkelmann, J., Schormair, B., Lichtner, P., Ripke, S., Xiong, L., Jalilzadeh, S., et al. (2007). Genome-wide association study of restless legs syndrome identifies common variants in three genomic regions. Nat. Genet. 39, 1000-1006. doi: $10.1038 /$ ng2099

Yang, Q., Li, L., Chen, Q., Foldvary-Schaefer, N., Ondo, W. G., and Wang, Q. K. (2011). Association studies of variants in MEIS1, BTBD9, and MAP2K5/SKOR1 with restless legs syndrome in a US population. Sleep Med. 12, 800-804. doi: 10.1016/j.sleep.2011.06.006

Yeomans, J. S., Li, L., Scott, B. W., and Frankland, P. W. (2002). Tactile, acoustic and vestibular systems sum to elicit the startle reflex. Neurosci. Biobehav. Rev. 26, 1-11. doi: 10.1016/s0149-7634(01)00057-4

Yun, S. P., Kim, D., Kim, S., Kim, S., Karuppagounder, S. S., Kwon, S. H., et al. (2018). $\alpha$-Synuclein accumulation and GBA deficiency due to L444P GBA 
mutation contributes to MPTP-induced parkinsonism. Mol. Neurodegener. 13:1. doi: 10.1186/s13024-017-0233-5

Zhang, J. Y., Liu, T. H., He, Y., Pan, H. Q., Zhang, W. H., Yin, X. P., et al. (2019). Chronic stress remodels synapses in an amygdala circuit-specific manner. Biol. Psychiatry 85, 189-201. doi: 10.1016/j.biopsych.2018.06.019

Zou, J., Pan, Y. W., Wang, Z., Chang, S. Y., Wang, W., Wang, X., et al. (2012). Targeted deletion of ERK5 MAP kinase in the developing nervous system impairs development of GABAergic interneurons in the main olfactory bulb and behavioral discrimination between structurally similar odorants. J. Neurosci. 32, 4118-4132. doi: 10.1523/jneurosci.6260-11.2012
Conflict of Interest: The authors declare that the research was conducted in the absence of any commercial or financial relationships that could be construed as a potential conflict of interest.

Copyright (C) 2021 Huang, Wang, Morales, Luo and Ma. This is an open-access article distributed under the terms of the Creative Commons Attribution License (CC BY). The use, distribution or reproduction in other forums is permitted, provided the original author(s) and the copyright owner(s) are credited and that the original publication in this journal is cited, in accordance with accepted academic practice. No use, distribution or reproduction is permitted which does not comply with these terms. 